\title{
Impact of Relay Stations Implementation on the Handover in WiMAX
}

\author{
Zdenek Becvar \\ Czech Technical University, Department of Telecommunication Engineering, Technicka 2, \\ 16627 Prague, Czech Republic \\ becvaz1@fel.cvut.cz
}

\begin{abstract}
Mobile WiMAX networks are generally based on the $802.16 \mathrm{e}$ standard. This version of standard supports a number of handover types and allows full mobility of users. However, the relay stations are not considered in this standard version. Relay stations are introduced in the originating $802.16 \mathrm{j}$ standard version. This paper deals with the impact of relay stations implementation from the handover point of view. The influences of fixed relay stations amount on the number of handover procedure initializations and on the diversity set size are investigated.
\end{abstract}

Keywords: WiMAX, Handover, Relay station, Diversity Set.

\section{Introduction}

Mobile WiMAX, based on 802.16e [1] standard, is a high-speed wireless networking communication system. WiMAX allows Non-Line-Of-Sight (NLOS) and Line-OfSight (LOS) communication in the frequency range $2-11 \mathrm{GHz}$ and $10-66 \mathrm{GHz}$ respectively. The physical layer in $802.16 \mathrm{e}$ uses SOFDMA (Scalable Orthogonal Frequency Division Multiple Access) to achieve high transfer speed. WiMAX allows large coverage up to $50 \mathrm{~km}$ for NLOS [2]. Further, the QoS support and hard and soft handovers to support full mobility are implemented in WiMAX.

The handover according to $802.16 \mathrm{e}$ allows to users high speed (up to $160 \mathrm{~km} / \mathrm{h} \mathrm{[3]}$ mobility) and it can provide continuous data flow for all applications.

WiMAX, such as described in [1], do not consider the Relay Stations (RS). The implementation of these stations into WiMAX networks is the target of just originating standard IEEE 802.16j [4]. The RS can be used to the coverage extension or to the BS's capacity increase. In this paper, the RSs placed to allow increasing of the capacity will be assume.

The rest of paper is organized as follows. Next section describes the handover types and handover process. The third section gives an overview about the RS and outlines possibilities to the implementation of the RSs to the networks. In the further section, the simulation parameters and scenarios are described. The fifth section contains the simulation results and their discussion. Last section presents our conclusions and future work plans. 


\section{Handover procedure in WiMAX}

The basic mean of the handover is to provide the continuous connection when a Mobile Station (MS) moves from an air-interface of one Base Station (BS) to an airinterface provided by another BS.

\subsection{Handover types}

IEEE 802.16e standard defines three basic types of handover [1], [5]: Hard Handover (HHO), Macro Diversity Handover (MDHO) and Fast Base Station Switching (FBSS). HHO is mandatory in WiMAX systems. Other two types of handover are optional. MDHO and FBSS can be called as the soft handovers.

Within hard handover, the MS communicates with just one BS in each time. All connections with old BS (called Serving BS) are broken before the connection to new BS (called Target BS) is established [5].

When the MDHO is supported by MSs and by BSs, the Diversity Set (in some publications noted as Active Set) is maintained by the MSs and by the BSs [5]. The Diversity Set is a list of the BSs, which are involved in the handover procedure. For downlink, one or more BSs from Diversity Set transmit data to the MS such that diversity combining can be performed at the MS. For uplink, the MS transmission is received by multiple BSs included in the Diversity Set and a selection diversity of received information is performed.

In the FBSS, the Diversity Set is maintained by the MS and by the BS similarly as in the MDHO. Opposite to the MDHO, the MS communicates only with an Anchor BS for all types of uplink and downlink traffic including management messages [5]. The Anchor BS is one of the BSs from Diversity Set in the MDHO. The MS is synchronized and registered to the Anchor BS; further the MS performs ranging and monitors the downlink channel for control information.

\subsection{Handover procedure}

The MS has to seek for suitable Neighbor BSs within normal operation mode to allow handover realization. Time dedicated for the searching for BSs in the neighborhood is assigned to the so-called scanning intervals. The MS determines the BS suitability to be a Target BS in these intervals. The scanning intervals are allocated via MAC management messages. After the MS finishes scanning of the Neighbor BSs, it sends the results to the Serving BS. There exist two types of results reporting. The information about used type is carried in the MOB_SCN-RSP and MOB_SCN-REP messages. In the first type, called Event trigger report, the MS reports based on the one of four defined trigger (CINR - Carrier to Noise plus Interface Ratio, RSSI Received Signal Strength Indication, Relative delay, RTD - Round Trip Delay). Measurement report is sent to the Serving BS after each measurement in this case. In the second type, called Periodic report, the measurement reports are sent at periodic intervals. The spacing of the reporting intervals is indicated in number of frames. The 
length of each frame can be up to $20 \mathrm{~ms}$. The number of frames between reports is contained in MOB_SCN-RSP message and the maximal number of frame is $256\left(2^{8}\right)$. So the maximal value can be set up to $5.12 \mathrm{~s}$. The handover should start immediately after the BS resolution based on the reported information.

\section{Relay station}

Relay stations are generally simplified kind of a BS. Two types of the RSs are defined: fixed and mobile. Fixed RS is permanently installed at the same place. Fixed and mobile RSs are connected to the network via radio interface and there is no connection to the wire backbone.

Wireless RS can be used to the coverage extension [6] or to increase of the capacity of the BSs [7]. The scenario with the RSs used for coverage extension is illustrated in Fig. 1. The MS communicates with the BS via two RSs. The RS is placed close to the boarder of the previous RS (or BS). The communication frame has to be divided on two parts [8]. In the first part, the RS serving local users (it functions as a BS). In the second part, the RS relays the data from the BS (or RS) to another RS (or BS).

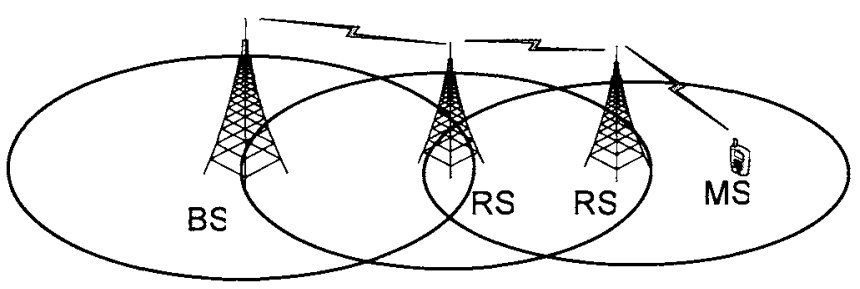

Fig. 1. Relay stations used to the enlarging of the coverage area.

Situation with the RS used to capacity increase is showen in Fig. 2. The frame has to be divided similary as in previous case. The difference to the area enlarging is in the placement of the the RS. In this case, the RS is situated into the area covered by the BS so that whole RS's coverage area is inside of the BS coverage area.

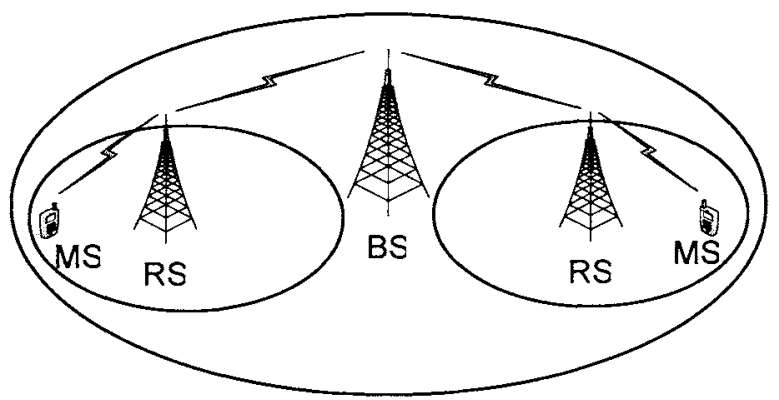

Fig. 2. Relay stations used to the increase of the capacity. 
Two concepts of the RSs implementation into WiMAX are considered [8]. The first type is a centralized system. The functionality of the RS is very simple in the centralized system. The RS only forwards received messages in direction to the target station. The RS is fully controlled by the BS. Therefore, the RS needs no special intelligence. The second concept is a distributed system. Opposite to the centralized system, the RS can modify management messages according to the situation in neighborhood. The RS in the distributed system must have implemented some controlling and management logic on the similar level as it is in the BS.

Relating to the handover management messages, there are not needed changes in the centralized system, but the creation of new management messages is necessary in the distributed system because the RS has to obtain information about neighborhood. These messages and their exchange are described in [9].

\section{Simulation scenario}

We can assume both soft handover types (MDHO and FBSS) because there are no differences in the Diversity Set and the Anchor BS updating procedures. We assume the measurement reports sent periodically with the reporting period length equal to $1 \mathrm{~s}$ ( $20 \mathrm{~ms}$ frame length and reporting after each 50 frames). The mobile users are moving by $50 \mathrm{~km} / \mathrm{h}$ speed. All systems operate in $5 \mathrm{GHZ}$ frequency band. All signal strengths among the MS and the BS (RS) are evaluated based on the Okumura-Hata path loss model for small or medium city [10]. This model (1) takes into account a distance between a MS and a BS (or RS) (in equation noted as $d$ ), height of BSs and RSs antennas $\left(h_{t}\right)$, transmitting frequency $(f)$ and height of MS $\left(h_{r}\right)$.

$$
\begin{gathered}
\mathrm{L}=69.55-13.82 * \log \mathrm{h}_{\mathrm{t}}-(1.1 * \log \mathrm{f}-0.7) * \mathrm{~h}_{\mathrm{r}}+(1.56 * \log \mathrm{f}-0.8) \\
+22.16 * \mathrm{f}+\left(44.9-6.55 * \log \mathrm{h}_{\mathrm{t}}\right) * \log \mathrm{d}
\end{gathered}
$$

Creating and updating of the Diversity Set is based on the principles described in [1]. Firstly, the BS (or RS) with the highest signal level (Anchor BS) is selected by each MS. Next, the Diversity Set is created for each MS depended on the defined thresholds and the signal strengths in the MS's location. All BSs and RSs with the signal strength higher than Add_Threshold (2) are added to the Diversity Set. The BS and RS can be also deleted from the Diversity Set, in the further step, if the signal level is lower than Delete_Threshold (3). Add and Delete thresholds are set up to 4 $\mathrm{dB}$ and $3 \mathrm{~dB}$ respectively.

$$
\text { BS signal level > Add_Threshold . }
$$

BS signal level $<$ Delete_Threshold .

Movement of the MSs is evaluated based on the Probabilistic Random Walk Mobility Model [11]. 
Four different scenarios were considered. The deployments of the MSs, BSs and RSs at the beginning of simulation for each scenario are shown in Fig. 1. Four BSs are placed on the same place near to the corners of simulated area in all scenarios. In the first scenario (scenario A), no RS is situated. In the second scenario (scenario B), only one RS in the middle of area is placed to improve signal conditions, because the signal level around the middle is week. In the third case (scenario C), $5 \mathrm{RS}$ are placed. One RS is placed in the middle, as in scenario B and each of the resting 4 RSs are situated between two Neighbor BSs. In the last case (scenario D), 9 RSs are placed. Five RSs is in the same location as in scenario C. Another 4 RSs are situated in the half way between BS and middle RS. The RSs in all scenarios are deployed to increase network throughput or number of connected user, but they do not enlarge the area. Fixed RSs are assumed. A movement of all MSs was same for all scenarios.

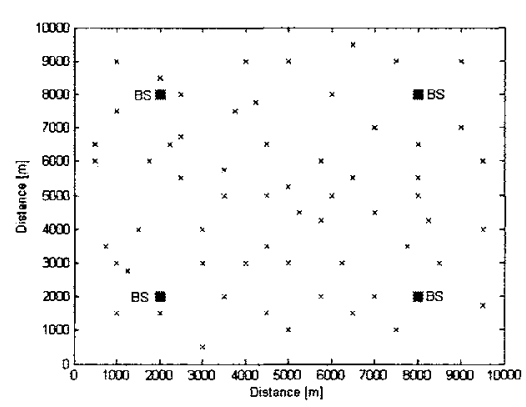

Scenario A

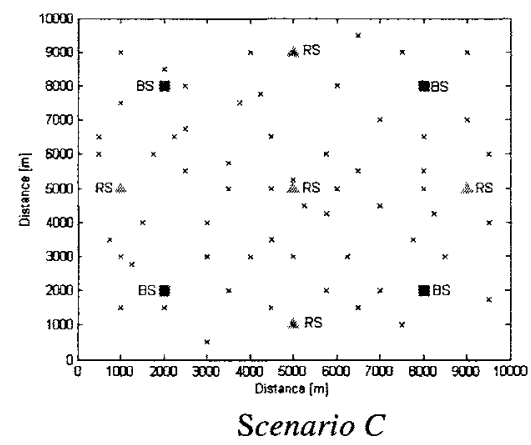

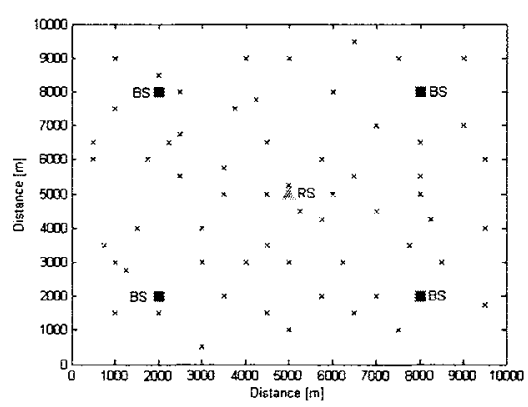

Scenario $B$

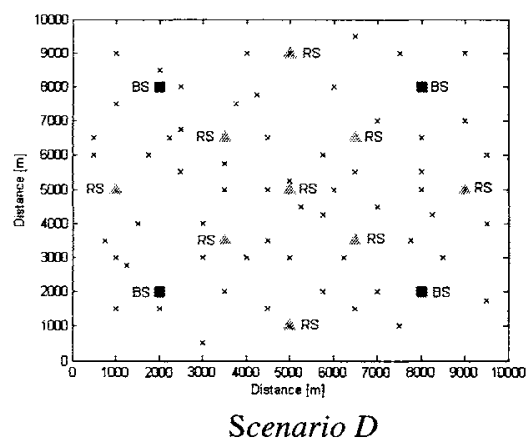

Fig. 3. Four cases of BSs (rectangles), fixed RSs (triangles) and MSs (crosses) deployment at the beginning of simulation. The scenario $A$ is scenario without RS, 1 RS is in scenario $B$, 5 RSs are in scenario $C$ and 9 RSs are in scenario $D$.

The MATLAB was used for the creation of simulation. Main parameters used in simulation are summarized in Table 1. 
Table 1. Simulation parameters.

\begin{tabular}{cc}
\hline Parameter name & Value \\
\hline Handover type & MDHO \\
Propagation model & Okumura-Hata suburban \\
Mobility Model & Probabilistic Random Walk \\
Size of simulated area & $10 \times 10 \mathrm{~km}$ \\
Number of MSs & 60 \\
Number of BSs & 4 \\
Number of RSs & $0 / 1 / / 5 / 9$ \\
BS transmitting power & $170 \mathrm{~dB}$ \\
BS's range radius & $4200 \mathrm{~m}$ \\
RS transmitting power & $146 \mathrm{~dB}$ \\
RS's range radius & $1000 \mathrm{~m}$ \\
Height of BSs and RSs & $30 \mathrm{~m}$ \\
Height of MSs & $2 \mathrm{~m}$ \\
Frequency & $5 \mathrm{GHz}$ \\
Speed of MSs & $50 \mathrm{~km} / \mathrm{h}$ \\
Delete threshold & $3 \mathrm{~dB}$ \\
Add threshold & $4 \mathrm{~dB}$ \\
Frame length & $20 \mathrm{~ms}$ \\
Reporting period & $50 \mathrm{frames}$ \\
Length of simulation & $900 \mathrm{~s}$ \\
Simulation step & $1 \mathrm{~s}$ \\
\hline
\end{tabular}

\section{Results}

Because the part of data flow is processed in the RSs, the capacity or the number of users can be increased when the RSs are added to the network [7]. The MS can communicate with more BSs (RSs) in the case of the MDHO at one time. In this case, the MS communicates (including data traffic) with all BSs (RSs) from Diversity Set. In the uplink, the MS sends the data to all BSs (RSs) and the selection diversity is performed. In the downlink, all BSs (RSs) send the data to the MS and the diversity combining is performed. The changes in the average number of the BSs and the RSs communicate with one MS at one time are shown in Fig.4. If we assume each MS connected to at least one BS or RS (it means each MS is connected to the network), so the average number of connection per MS has to be equal or greater then 1; and the total number of connections in whole area at one time have to be equal or grater then a number of the MSs in the area. The independence of the average number of connections to the BSs (average number of BSs in the Diversity Set) on the count of RSs in area is caused by using of the absolute threshold (according to [1]). The BSs are added to the Diversity Set independent on the signal level of the other BSs or RSs. The increase of the average number of connections to the RSs (average number of the 
RSs in the Diversity Set) is depending on the number of the RSs in the area. The size of the Diversity Set is equal to the average number of connections to the BSs plus the average number of connections to the RSs. Therefore the size of the Diversity Set is increasing with placing of the others RSs to the network.

In the FBSS, the situation is different. There, the MS exchanges user data with only one Anchor BS or RS at one time. Therefore, the total amount of BSs (or RSs) connected to the one MS is still same and is equal to the number of MSs in network.

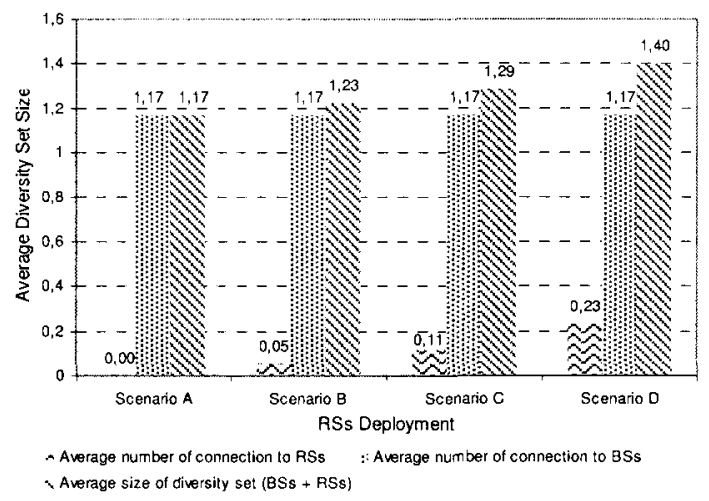

Fig. 4. The average number of connections at one time among MS and BSs (bar filled with dot), among MS and RSs (bar filled with wavelet) and among MS and BSs plus RSs (bar filled with back slash lines) per one MS.

Similar to this, with the increasing amount of the RSs in the area, the number of initialization of the handover procedures (in this case, handover procedures mean booth Diversity Set update and Anchor BS update) is rising too (see Fig.5). It is caused by increasing the area where the handover is initialized (the places where a signal level from BSs or RSs is higher than selected thresholds). Hence, users move across these areas more often. This results in an increasing number of handover messages exchanged in the network.

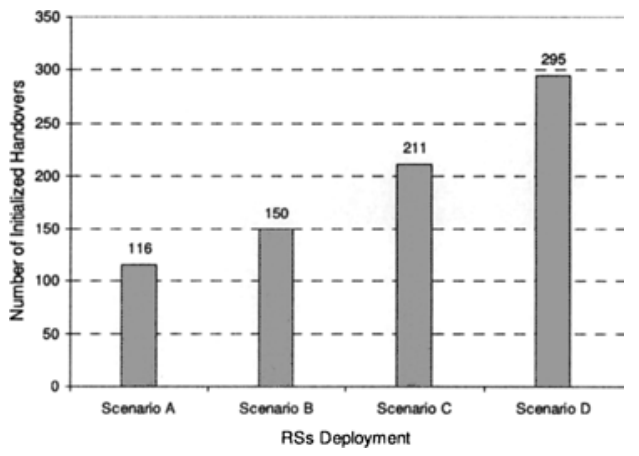

Fig. 5. Number of initialized handovers during simulation depending on RS deployment. 


\section{Conclusions and future work plans}

We have investigated an impact of the Diversity Set size and an impact of amount of the handover procedure initialization on the number of RSs. The RSs location was selected based on the signal condition. It means that the RSs were placed into the areas with worst signal level, to increase throughput in this area.

The average number of BSs in the Diversity Set is independent on the count of the RSs in area if we assume absolute thresholds. Opposite to this, the average amount of the RSs in the Diversity Set is increasing with growing number of the RSs in the area. Therefore, average size of the Diversity Set (including BSs and RSs) is increasing to. The increase of the Diversity Set size results to the raising of the number of initialization of the handover procedure.

Increase of the handover initializations and rising of the Diversity Set size brings more frequent management messages exchange and decrease the user's data throughput.

In the future, we will compare the Diversity Set size for absolute thresholds and relative thresholds and next, we will investigate an impact of the RSs placement on the ratio between user's and management data.

Acknowledgments. This research work was supported by Czech Technical University's grant No. CTU0715013.

\section{References}

1. IEEE P802.16e/D12: Air Interface for Fixed and Mobile Broadband Wireless Access Systems: Amendment for Physical and Medium Access Control Layers for Combined Fixed and Mobile Operation in Licensed Bands. New York (2005)

2. WiMAX Forum: WiMAX's technology for LOS and NLOS environments. (2004)

3. WiMAX Forum: Can WiMAX Address Your Applications?. (2005)

4. IEEE 802.16's Relay Task Group: IEEE 802.16j proposals. <http://www.ieee802.org/16/relay/>

5. Becvar, Z., Zelenka, J., : Implementation of Handover Delay Timer into WiMAX. 6th Conference on Telecommunication. Peniche, Portugal (2007)

6. Wei, Z. : Capacity Analysis for Multi-hop WiMAX Relay

7. Mach, P., Bestak, R.: Performance of IEEE 802.16 Enhanced by Relay Stations. 6th Conference on Telecommunication. Peniche, Portugal (2007)

8. Hoymann, Ch., Klagges K.: MAC frame concepts to support multihop communication in IEEE 802.16 Networks. Wireless World Research Forum, Shanghai, China (2007)

9. Lee, H., Wong, W. C., Jerry, S., Johnsson, K., Yang, S., Lee, M.: Overview of the proposal for MS MAC handover procedure in an MR Network. IEEE 802.16j proposal, No. S80216j06_217r1.pdf (2007)

10.Korowajczuk, L., Souza, B., Xavier, A., Moreira, A., Filho, F., Ribeiro, L. Z., Korowajczuk, C., DaSilva, L. A.: Designing CDMA 2000 Systems, Wiley, Great Britain (2004)

11.T. Camp, J. Boleng, V. Davies, "A Survey of Mobility Models for Ad Hoc Network Research. (2002) 